\title{
MASS ESTIMATORS FOR FLATTENED DISPERSION-SUPPORTED GALAXIES
}

JASON L. SANDERS AND N. WYN EVANS

Institute of Astronomy, Madingley Road, Cambridge CB3 0HA, UK; jls@ast.cam.ac.uk, nwe@ast.cam.ac.uk Received 2016 August 26; revised 2016 September 16; accepted 2016 September 18; published 2016 October 12

\begin{abstract}
We investigate the reliability of mass estimators based on the observable velocity dispersion and half-light radius $R_{\mathrm{h}}$ for dispersion-supported galaxies. We show how to extend them to flattened systems and provide simple formulae for the mass within an ellipsoid under the assumption the dark-matter density and the stellar density are stratified on the same self-similar ellipsoids. We demonstrate explicitly that the spherical mass estimators give accurate values for the mass within the half-light ellipsoid, provided $R_{\mathrm{h}}$ is replaced by its "circularized" analog $R_{\mathrm{h}} \sqrt{1-\epsilon}$. We provide a mathematical justification for this surprisingly simple and effective workaround. It means, for example, that the mass-to-light ratios are valid not just when the light and dark matter are spherically distributed, but also when they are flattened on ellipsoids of the same constant shape.
\end{abstract}

Key words: dark matter - galaxies: dwarf - galaxies: kinematics and dynamics

\section{INTRODUCTION}

Accurate estimates of the dark-matter content of dwarf spheroidal galaxies (dSphs) are crucial for furthering our understanding of galaxy formation and structure. Calculating reliable mass estimates has historically been an awkward problem as, with only line of sight (LOS) velocity measurements, the mass profile of a spherical galaxy can only be inferred by making an assumption about the degree of velocity anisotropy, i.e., the ratio of radial to tangential motion.

Through comparisons to solutions of the Jeans equations, it has been shown that the mass contained near the half-light radius of a dispersion-supported galaxy is approximately independent of the velocity anisotropy and the radial profile of the dark and luminous matter and is simply related to the half-light radius $R_{\mathrm{h}}$ and the luminosity-averaged LOS velocity dispersion $\sqrt{\left\langle\sigma_{\text {los }}^{2}\right\rangle}$. There exist several different forms for these formulae in the literature (Walker et al. 2009; Wolf et al. 2010; Amorisco \& Evans 2012; Campbell et al. 2016) that may be summarized as

$$
M_{\mathrm{sph}}\left(<r_{x}\right)=\frac{C_{x}\left\langle\sigma_{\text {los }}^{2}\right\rangle R_{\mathrm{h}}}{G}
$$

where $M_{\mathrm{sph}}\left(<r_{x}\right)$ is the mass contained within a sphere of radius $r_{x}$ and $G$ the familiar gravitational constant. $C_{x}$ is a constant that depends on the choice of radius $r_{x}$. Walker et al. (2009) proposed that if $r_{x}=R_{\mathrm{h}}$, then $C_{x}=2.5$ based on a simple example of the stellar distribution following a Plummer profile and the dark matter following a cored isothermal profile, although this was validated through fuller testing. Wolf et al. (2010) demonstrated that for $r_{x} \approx \frac{4}{3} R_{\mathrm{h}}$ (approximately the 3D spherical half-light radius for a range of observationally motivated profiles) that $C_{x}=4$ reproduced the results from full Jeans analyses and was also shown to be mathematically true under the assumption of a near-flat velocity dispersion profile.

Although spherical mass estimators have proved useful for understanding dSphs, they cannot give the full picture as they do not consider the fundamentally aspherical shape of these galaxies. Our aim in this Letter is to find mass estimators equivalent to Equation (1) applicable to flattened systems. We begin by inspecting the validity of the spherical mass estimators and go on to investigate the applicability of the estimator when considering flattened systems in which the dark and light matter are stratified on the same self-similar ellipsoids. We give formulae similar to Equation (1) that may be used when the 3D shape of the system is known. By marginalizing over prior assumptions on the intrinsic shape and alignment, we show how the mass can be estimated when the intrinsic shape and alignment are not known.

\section{SPHERICAL MASS ESTIMATORS}

For a spherical stellar luminosity density $j_{\star}(r)$ with a constant mass-to-light ratio in a spherical mass density $\rho_{\mathrm{DM}}(r)$ with mass profile $M(r)$ sourcing potential $\Phi(r)$, the potential energy can be written in terms of the surface brightness $S(R)$ as

$$
W=\frac{1}{2} \int d V j_{\star}(r) \Phi(r)=4 \pi G \int_{0}^{\infty} d r I(r) M^{\prime}(r),
$$

where

$$
I(r)=\int_{r}^{\infty} d r r j_{\star}(r)=-\frac{1}{\pi} \int_{r}^{\infty} d R\left(R^{2}-r^{2}\right)^{1 / 2} \frac{d S}{d R} .
$$

From the virial theorem, we know that the LOS velocity dispersion is related to the total luminosity $L$ by $\left\langle\sigma_{\text {los }}^{2}\right\rangle=-W / 3 L$, which gives the constant $C_{x}$ as

$$
C_{x}=\frac{1}{R_{\mathrm{h}}}\left[\int_{0}^{r_{x}} d r r^{2} \rho_{\mathrm{DM}}(r)\right]\left[\int_{0}^{\infty} d r r^{2} J(r) \rho_{\mathrm{DM}}(r)\right]^{-1}
$$

where $J(r)=(4 \pi / 3 L) I(r)$. The constant $C_{x}$ depends only on the profile of the halo model $\rho_{\mathrm{DM}}$ and the surface brightness profile $J(r)$.

We use this to test the validity of the spherical mass estimator. In Figure 1, we show the result of Equation (4) computed numerically for two models with differing ratios of dark to stellar scale-lengths $\left(r_{\mathrm{DM}} / R_{\mathrm{h}}\right)$. They are an NFW darkmatter profile $\rho_{\mathrm{DM}}(r) \propto r^{-1}\left(1+r / r_{\mathrm{DM}}\right)^{-2}$ and a cored isothermal profile of the form

$$
\rho_{\mathrm{DM}}(r)=\frac{v_{0}^{2}}{4 \pi G} \frac{3 r_{\mathrm{DM}}^{2}+r^{2}}{\left(r_{\mathrm{DM}}^{2}+r^{2}\right)^{2}} .
$$




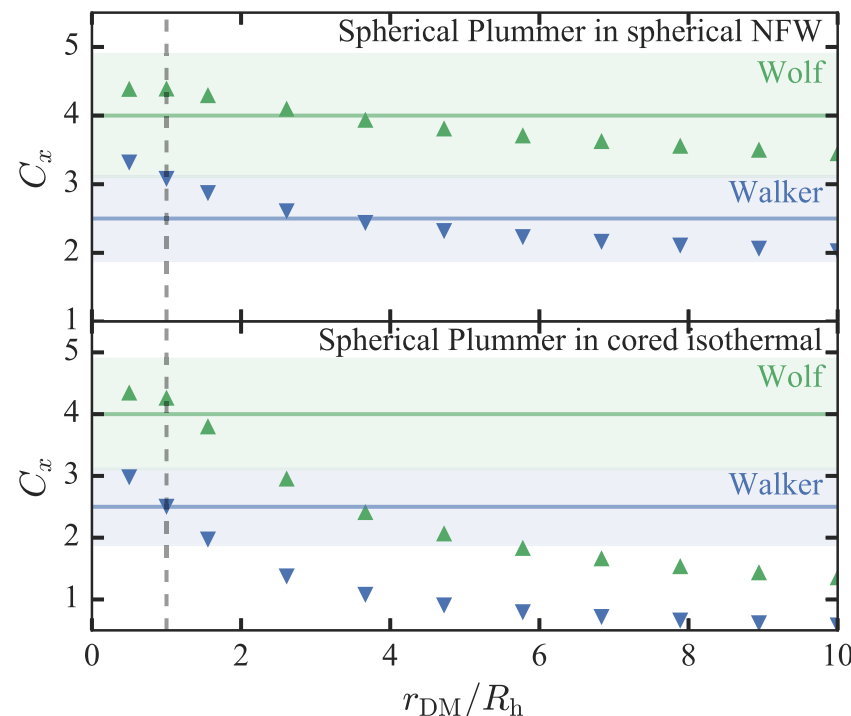

Figure 1. Constant $C_{x}$ in the spherical mass estimator formula against the ratio of the dark-matter scale radius to the stellar half-light radius for a Plummer model embedded in an NFW (upper panel) and cored isothermal (lower) halo. The blue inverted triangles show $C_{x}$ at the radius $r=R_{\mathrm{h}}$, for which Walker et al. (2009) advocate a value of $C_{x}=2.5$ (shown with a solid blue horizontal line). The green triangles show the $C_{x}$ at the radius $r=\frac{4}{3} R_{\mathrm{h}}$, for which Wolf et al. (2010) advocate a value of $C_{x}=4$ (green line). The bands show the uncertainties from Campbell et al. (2016).

The stellar tracer profile follows a Plummer law $\rho_{\star}(r) \propto\left(1+\left(r / r_{\star}\right)^{2}\right)^{-5 / 2}$ for which $R_{\mathrm{h}}=r_{\star}$. The constant $C_{x}$ is computed at the two radii recommended by Walker et al. (2009) and Wolf et al. (2010). The constants given by these two authors are shown with horizontal lines along with the uncertainty found by Campbell et al. (2016) from inspecting cosmological hydrodynamical simulations. The variation of $C_{x}$ with respect to $r_{\mathrm{DM}} / R_{\mathrm{h}}$ is smallest for the NFW profile and is consistent with the bracket found by Campbell et al. (2016). In the cored isothermal profile with $r_{\mathrm{DM}} / R_{\mathrm{h}} \approx 1$, both estimators perform well. However, again, as $r_{\mathrm{DM}} / R_{\mathrm{h}}$ is increased, $C_{x}$ deviates significantly and so the estimators perform poorly for $r_{\mathrm{DM}} / R_{\mathrm{h}}>2$.

We now explore how the mass estimators perform as the parameters of a double power-law dark-matter density profile are altered. We use a fixed Plummer profile for the stars with a sech truncation at $10 R_{\mathrm{h}}$. In Figure 2, we show the mass profiles of different dark-matter profiles that all produce the same luminosity-averaged LOS velocity dispersion. The default parameters are those of an NFW profile with $r_{\mathrm{DM}} / R_{\mathrm{h}}=1$ and a sech truncation at $10 r_{\mathrm{DM}}$. We alter the outer slope $\beta$, inner slope $\gamma$, and the ratio $r_{\mathrm{DM}} / R_{\mathrm{h}}$. We find that when varying the inner and outer slopes the pinch point where the mass is the same for all profiles is around $\frac{4}{3} R_{\mathrm{h}}$, i.e., the radius recommended by Wolf et al. (2010). Varying $r_{\mathrm{DM}} / R_{\mathrm{h}}$ produces a pinch point further out. This helps explain why mass estimators derived for use on realistic halos with $r_{\mathrm{DM}}>R_{\mathrm{h}}$ can constrain the mass at larger radii (e.g., Amorisco \& Evans 2012; Campbell et al. 2016).

\section{FLATTENED MASS ESTIMATORS}

We now turn to adapting the spherical mass estimators for application to flattened systems. We work with models with both the dark and stellar density stratified on the same concentric self-similar ellipsoids labeled with the coordinate $m$ such that $m^{2}=x^{2} / a^{2}+y^{2} / b^{2}+z^{2} / c^{2}$ with $a>b>c$. The axis ratios of the ellipsoids are $p=b / a$ and $q=c / a$. We view the model along the spherical polar unit vector defined by the angles $(\vartheta, \varphi)$, where $\vartheta$ is the co-latitudinal angle and $\varphi$ the azimuthal angle defined with respect to a Cartesian coordinate system aligned with the principal axes (see Figure 3). When oblate and prolate spheroids are viewed "face-on," they appear round. The spherical mass estimator underestimates (overestimates) the mass within a sphere for the oblate (prolate) case, as mass is added to (removed from) the sphere. Similarly, the formulae give (smaller) under- and overestimates for the mass within the corresponding ellipsoid. We seek an appropriate modification to Equation (1) that is applicable to flattened systems, namely,

$$
M_{\mathrm{ell}}\left(<m_{x}\right)=\frac{C_{x} f_{\sigma}\left\langle\sigma_{\mathrm{los}}^{2}\right\rangle f_{r} R_{\mathrm{h}}}{G},
$$

where $M_{\text {ell }}\left(<m_{x}\right)$ is the mass within an ellipsoid (that is the same shape as the equidensity contours) with major axis length $r_{x}$. We imagine creating an ellipsoidal model by deforming a spherical model that obeys the spherical mass estimator formulae outlined in the previous section. The total mass is conserved if $a b c=1$ and the mass within an ellipsoid of major-axis length $r_{x}$ is identical to the mass within a sphere of radius $m_{x}=r_{x} / a=r_{x}(p q)^{1 / 3}$. However, to estimate this parent spherical model mass from the spherical mass estimator formulae, we must relate the observed LOS velocity dispersion to the spherical velocity dispersion and the observed half-light major-axis length to the intrinsic majoraxis length of the considered ellipsoid. Assuming the total velocity dispersion (the average of the dispersions along the principal axes) is conserved as we deform the model ${ }^{1}$, the factor $f_{\sigma}$ accounts for the relationship between the LOS velocity dispersion and the total dispersion of the ellipsoidal model. The factor $f_{r}$ accounts for the relationship between the observed major-axis length and the intrinsic major-axis length of the equivalent ellipsoid (and that of the parent spherical model).

\subsection{Velocity Scaling}

For triaxial systems, the velocity scaling $f_{\sigma}=\left\langle\sigma_{\text {tot }}^{2}\right\rangle /\left\langle\sigma_{\text {los }}^{2}\right\rangle$ is given by

$$
f_{\sigma}=\frac{1}{3} \frac{1+r_{x z}+r_{y z}}{\cos ^{2} \vartheta+r_{x z} \sin ^{2} \vartheta \cos ^{2} \varphi+r_{y z} \sin ^{2} \vartheta \sin ^{2} \varphi}
$$

where

$$
r_{i j}=\left\langle\sigma_{i}^{2}\right\rangle /\left\langle\sigma_{j}^{2}\right\rangle=W_{i i} / W_{j j} \text { (no sum). }
$$

For dSphs in which the stellar and dark-matter density profiles are stratified on the same self-similar ellipsoids, $r_{i j}$ depends only on the shape of the ellipsoids (Roberts 1962; Binney \& Tremaine 2008). That is to say, it is independent of the "radial" density profile of the light and dark matter. Therefore, $f_{\sigma}$ is a

\footnotetext{
1 To leading order in the flattening, the ratio of the total dispersion of the flattened model to the spherical model with the same mass is $\left\langle\sigma_{\text {tot }}^{2}\right\rangle_{\text {flat }} /\left\langle\sigma_{\text {tot }}^{2}\right\rangle_{\text {sph }} \approx 1-\frac{4}{45}\left[(1-p)^{2}-(1-p)(1-q)+(1-q)^{2}\right]$.
} 

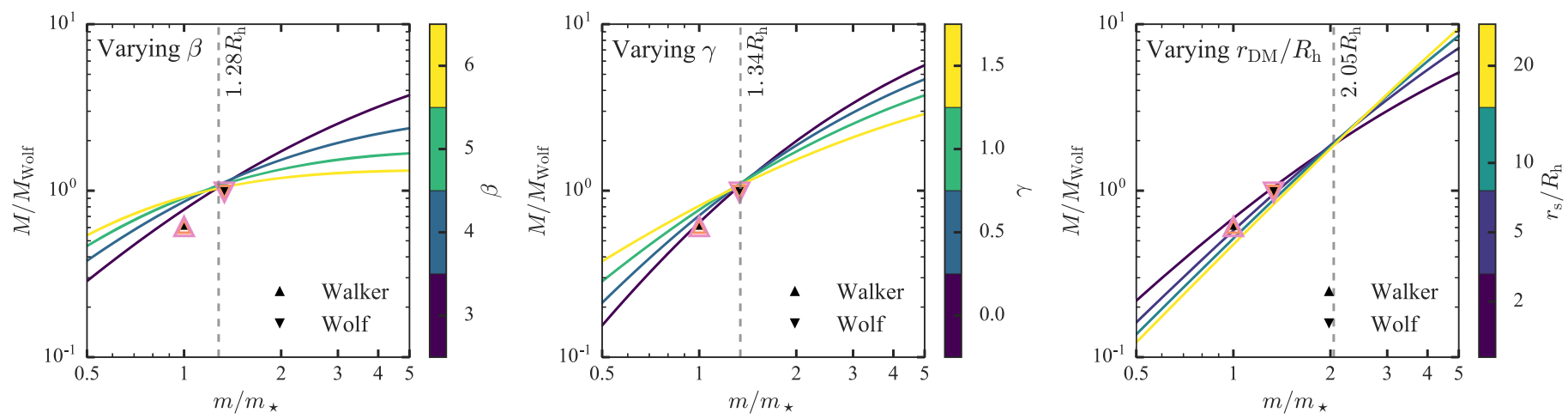

Figure 2. Spheroidal mass profile for a stellar Plummer profile embedded in a double power-law dark-matter halo with varying outer slope $\beta$, inner slope $\gamma$, and scale radius $r_{\mathrm{DM}}$. All models have the same luminosity-averaged LOS velocity dispersion and half-light radius $R_{\mathrm{h}}$. The masses are normalized with respect to the Wolf mass estimate. The default parameters are $\gamma=1, \beta=3$ (NFW), and $r_{s} / R_{\mathrm{h}}=1$. The black points show the results of two mass estimators and the vertical dashed line shows the point of minimum variance in the logarithm of the mass for each set of curves. The spheroidal mass estimates using the mass estimator proposed in this Letter are given for an edge-on oblate ( $q=0.6$, orange triangles) and edge-on prolate model ( $p=q=0.6$, pink triangles).
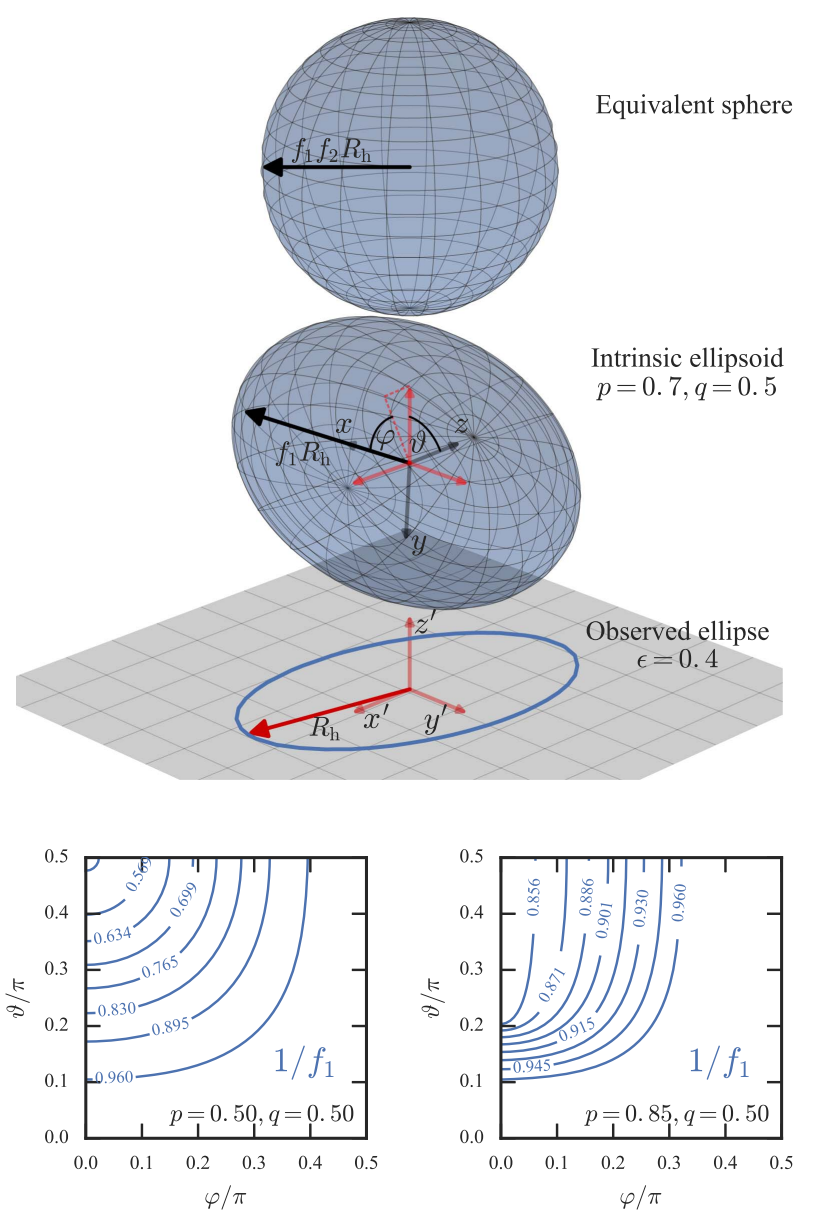

Figure 3. Relationship between the observed half-light radius and the radius used in our mass estimator formula. An ellipsoid is observed at spherical polar angles $(\vartheta, \varphi)$ with respect to its intrinsic Cartesian coordinates $(x, y, z)$ aligned with the principal axes. The resulting projection is an ellipse (shown below) with major-axis length $R_{\mathrm{h}}$ that lies in the $\left(x^{\prime}, y^{\prime}\right)$ plane of the observed Cartesian coordinate system $\left(x^{\prime}, y^{\prime}, z^{\prime}\right)$. Above the ellipsoid, we show the sphere with the equivalent volume as the ellipsoid. The major axis of the ellipse is related to the major axis of the ellipsoid by the factor $1 / f_{1}$, which is shown in the lower two panels for a prolate spheroid with $p=q=0.5$ (left) and an ellipsoid with axis ratios $p=0.85$ and $q=0.5$ (right).

function of $p, q$, and the viewing angles: $f_{\sigma}=f_{\sigma}(\vartheta, \varphi, p, q)$. Expressions for $W_{i j}$ are given in Table 2.2 of Binney \& Tremaine (2008).

\subsection{Radial Scaling}

We decompose the radial scaling into two components, $f_{r}=f_{1} f_{2} . f_{2}$ describes the relationship between the ellipsoidal major-axis length and the parent spherical radius so (as described above) $f_{2}=(p q)^{1 / 3}$. The other factor $f_{1}$ gives the relationship between the observed major-axis length of the half-light ellipse $R_{\mathrm{h}}$ and the intrinsic major-axis length of the corresponding ellipsoid $r_{\text {maj. }}$. In the spherical case, these quantities are equal. In the ellipsoidal case, the relationship between these quantities depends on the viewing angles and the intrinsic shape $f_{1}=f_{1}(\vartheta, \varphi, p, q)$. We approximate $f_{1}$ by the relationship between the major-axis length of an ellipsoid and the major-axis length of its projected ellipse. This neglects any subtleties related to the extended nature of the true density distribution. However, if the 3D stellar light profile falls off sufficiently rapidly, then our relationship is a good approximation.

To derive our approximation for $f_{1}$, we use a coordinate system $\left(x^{\prime}, y^{\prime}, z^{\prime}\right)$ related to the intrinsic coordinate system by (see Figure 3)

$$
\begin{aligned}
& x=-x^{\prime} \sin \varphi-y^{\prime} \cos \vartheta \cos \varphi+z^{\prime} \sin \vartheta \cos \varphi \\
& y=x^{\prime} \cos \varphi-y^{\prime} \cos \vartheta \sin \varphi+z^{\prime} \sin \vartheta \sin \varphi \\
& z=y^{\prime} \sin \vartheta+z^{\prime} \cos \vartheta
\end{aligned}
$$

We consider the set of points where the ellipsoidal surface is tangential to $\hat{z}^{\prime}$ which results in a rotated ellipse in the $\left(x^{\prime}, y^{\prime}\right)$ plane. We diagonalize the resultant quadratic surface to find the major axis length $R_{\mathrm{h}}$ as

$$
f_{1}^{-2}=\left(R_{\mathrm{h}} / r_{\mathrm{maj}}\right)^{2}=2 C /(A-\sqrt{B}),
$$

where

$$
\begin{aligned}
A= & \left(1-q^{2}\right) \cos ^{2} \vartheta+\left(1-p^{2}\right) \sin ^{2} \vartheta \sin ^{2} \varphi+p^{2}+q^{2}, \\
B= & {\left[\left(1-q^{2}\right) \cos ^{2} \vartheta-\left(1-p^{2}\right) \sin ^{2} \vartheta \sin ^{2} \varphi-p^{2}+q^{2}\right]^{2} } \\
& +4\left(1-p^{2}\right)\left(1-q^{2}\right) \sin ^{2} \vartheta \cos ^{2} \vartheta \sin ^{2} \varphi, \\
C= & p^{2} \cos ^{2} \vartheta+q^{2} \sin ^{2} \vartheta\left(p^{2} \cos ^{2} \varphi+\sin ^{2} \varphi\right) .
\end{aligned}
$$

As given in Weijmans et al. (2014), the observed ellipticity $\epsilon$ satisfies $(1-\epsilon)^{2}=(A-\sqrt{B}) /(A+\sqrt{B})$. 
For an oblate spheroid ( $p=1$ ), Equation (10) simplifies to $R_{\mathrm{h}}=r_{\text {maj. }}$. For a prolate spheroid $p=q$, so we find

$$
f_{1}^{-2}=\cos ^{2} \vartheta+\sin ^{2} \vartheta\left(q^{2} \cos ^{2} \varphi+\sin ^{2} \varphi\right) .
$$

In Figure 3, we show the major axis length for a prolate figure and a triaxial figure as a function of the viewing angle.

The ellipsoidal half-light radius $m_{h}$ is well approximated by ${ }_{3}^{4} f_{r} R_{\mathrm{h}}$, which should be compared to the radius of $\frac{4}{3} R_{\mathrm{h}} \sqrt{1-\epsilon}$ that is empirically used (e.g., Koposov et al. 2015; Sanders et al. 2016) as $R_{\mathrm{h}} \sqrt{1-\epsilon}$ approximately reproduces the circularly averaged half-light radius of the $\mathrm{dSph} .^{2}$

\subsection{Near-spherical Limits}

Using Equation (10), we can find the modification factor $f_{\sigma} f_{r}$ for the simple cases of viewing down the principal axes of a near-spherical triaxial ellipsoid and compare to the alternative factor $\sqrt{1-\epsilon}$. When viewing down the major axis $(\vartheta=\pi / 2, \varphi=0)$, we find

$$
f_{\sigma} f_{r} \approx 1+\frac{2}{5}(1-p)-\frac{3}{5}(1-q) .
$$

The observed ellipticity $\epsilon=1-p / q$ so the circularized factor $\sqrt{1-\epsilon} \approx 1+\frac{1}{2}(1-p)-\frac{1}{2}(1-q)$, which is a close approximation to our factor $f_{\sigma} f_{r}$. Similarly, for viewing down the intermediate axis, we find

$$
f_{\sigma} f_{r} \approx 1+\frac{1}{5}(1-p)-\frac{3}{5}(1-q),
$$

while $\sqrt{1-\epsilon}=\sqrt{q} \approx 1-\frac{1}{2}(1-q)$. Finally, viewing down the minor axis, we find

$$
f_{\sigma} f_{r} \approx 1-\frac{3}{5}(1-p)+\frac{1}{5}(1-q),
$$

while $\sqrt{1-\epsilon}=\sqrt{p} \approx 1-\frac{1}{2}(1-p)$. We note that the flattening in the LOS direction (e.g., $p$ in the intermediate axis case) has a smaller contribution to the factor $f_{\sigma} f_{r}$. This demonstrates that the simple factor $\sqrt{1-\epsilon}$ goes a long way to account for the velocity and radial scalings we propose.

\subsection{Results}

In Figure 2, we show the mass estimates using our formulae for an oblate and prolate model viewed edge-on. The models have the same ellipsoidal mass profile as the spherical model shown. The factors we have introduced correctly deproject the observed quantities producing an unbiased mass estimate. Figure 4 shows the constant in the half-light ellipsoid mass estimator (Equation (6)) for three models of flattened Plummer profiles embedded in equivalently flattened NFW halos with $m_{\mathrm{DM}} / m_{\star}=5$. We show an oblate, prolate, and triaxial $p=\frac{1}{2}(1+q)$ model. Simply using the spherical mass estimator with $R_{\mathrm{h}}$ underestimates/overestimates the ellipsoidal mass for the oblate/prolate case viewed face-on (down the minor/major axis). Similarly, the edge-on case (major for oblate, minor for prolate) produces overestimates of the mass for both oblate and prolate models. For the triaxial model the

\footnotetext{
2 For example, a flattened $(q=1-\epsilon)$ Plummer surface profile produces a circularly averaged half-light radius equal to $R_{\mathrm{h}} \sqrt{\frac{1}{6}\left(1+q^{2}+\sqrt{1+14 q^{2}+q^{4}}\right)}$, which for small flattenings is $R_{\mathrm{h}}\left(1-\frac{1}{2} \epsilon+\mathcal{O}\left(\epsilon^{4}\right)\right)$ so well approximated by $R_{\mathrm{h}} \sqrt{1-\epsilon}$.
}

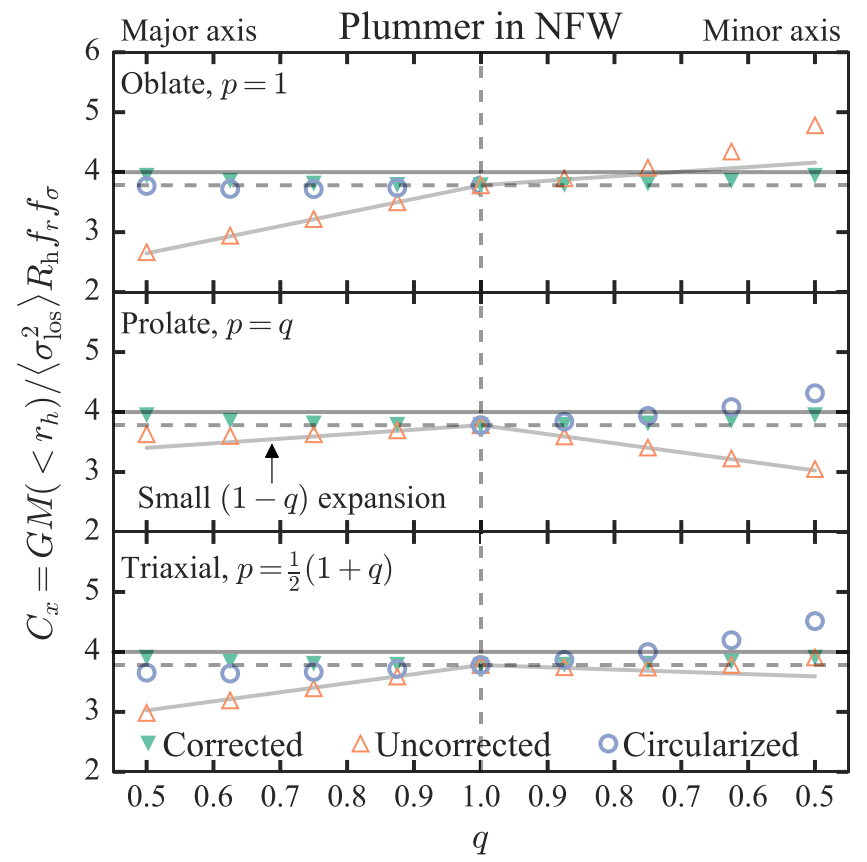

Figure 4. Mass estimator constant for the half-light ellipsoid against the flattening $q$ for Plummer models embedded in equivalently flattened NFW halos. The top panel shows an oblate model, the middle panel a prolate model, and the bottom panel a triaxial model with $p=\frac{1}{2}(1+q)$. The left half of the plot corresponds to viewing down the major axis while the right half corresponds to viewing down the minor axis. The corrected green filled triangles show the constant from the mass estimator formula given in this Letter, the uncorrected orange empty triangles show the constant using the spherical mass estimator (i.e., $f_{r} f_{\sigma}=1$ ), and the purple circles show the constant using the spherical mass estimator with the circularized radius $R_{\mathrm{h}} \sqrt{1-\epsilon}$ (not shown in the panels where $\epsilon=0$ ). The horizontal solid line shows the Wolf et al. constant and the dashed line shows the constant from the spherical model for this exact case. The other gray solid lines show the small $(1-q)$ expansion of $C_{x} f_{\sigma} f_{r}$ (i.e., the uncorrected constant).

spherical mass estimator produces an overestimate when viewing down the major axis and (for this particular case) is largely unbiased when viewing down the minor axis. The results using the correction factors $f_{\sigma}$ and $f_{r}$ are unbiased estimates of the mass within the ellipsoid $m=\frac{4}{3} m_{\star}$ and using the spherical mass estimator with the "circularized" radius $R_{\mathrm{h}} \sqrt{1-\epsilon}$ produces very similar results to the corrected version. This echoes a result in Sanders et al. (2016), who demonstrated that the correction to the $D$-factor (important for interpreting dark-matter decay signals) is almost independent of the flattening for edge-on systems. The near-spherical expansions of Section 3.3 are also shown, which replicate the trends over the full $q$ range.

Our proposed modifications correctly reproduce the mass within ellipsoids. However, this relies on knowing the intrinsic shape and alignment of the dSph. Such information is not accessible, but we can put priors on possible models that reproduce the observables. We choose to put priors on the triaxiality $T=\left(1-p^{2}\right) /\left(1-q^{2}\right)$, flattening $q$, and the viewing angles $(\vartheta, \varphi)$. We consider three priors:

1. Flat prior- $T \sim \mathcal{U}(0,1), q \sim \mathcal{U}(0.05,1), \cos \vartheta \sim \mathcal{U}(0$, 1), $\varphi \sim \mathcal{U}(0, \pi / 2)$.

2. Major-axis prior- $T \sim \mathcal{U}(0,1), \quad q \sim \mathcal{U}(0.05,1), \quad \vartheta \sim$ $\mathcal{N}(\pi / 2,0.1 \mathrm{rad}), \varphi \sim \mathcal{U}(0,0.1 \mathrm{rad})$. 


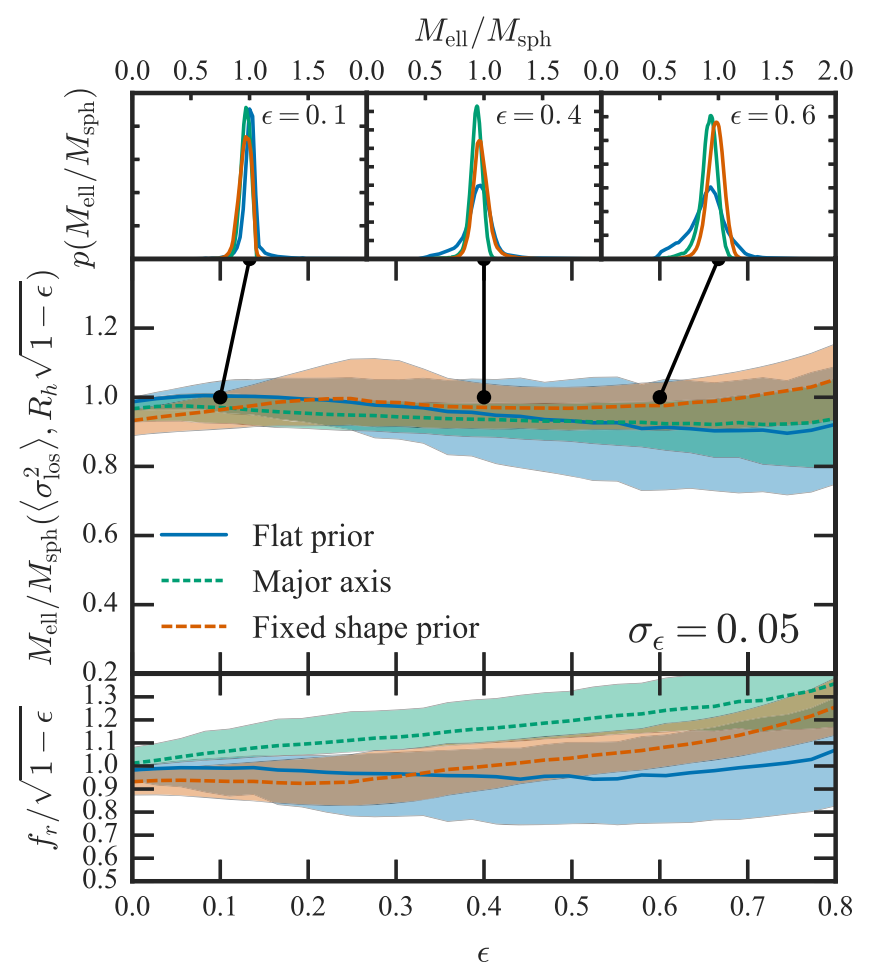

Figure 5. Ratio of the mass within the half-light ellipsoid to the mass estimated from the spherical mass estimator (Walker et al. 2009; Wolf et al. 2010) using the circularized half-light radius $R_{\mathrm{h}} \sqrt{1-\epsilon}$ against ellipticity. At each ellipticity, we show the median and $\pm 1 \sigma$ spreads in the mass estimates for triaxial figures within a 0.05 spread of the required ellipticity and with fixed $R_{\mathrm{h}}$ and $\left\langle\sigma_{\text {los }}^{2}\right\rangle^{1 / 2}$ under three prior assumptions: the blue solid line corresponds to a uniform prior on viewing angle, intrinsic ellipticity, and triaxiality; the green short-dashed line corresponds to a prior of preferentially viewing down the major axis; and the orange long-dashed line corresponds to a fixed triaxiality and intrinsic ellipticity prior. The three histograms show slices through the main figure at $\epsilon=0.1,0.4$ and 0.6. The lower panel shows the median and $\pm 1 \sigma$ brackets of $f_{r} / \sqrt{1-\epsilon}$ - the ratio of the dSph radius to the "circularized" radius.

3. Fixed-shape prior- $T \sim \mathcal{N}(0.55,0.04), \quad q \sim \mathcal{N}(0.49$, $0.12), \cos \vartheta \sim \mathcal{U}(0,1), \varphi \sim \mathcal{U}(0, \pi / 2)$.

The final prior is taken from a fit to the shapes of the Local Group dSphs from Sánchez-Janssen et al. (2016). The majoraxis prior is inspired by the observation from simulations that the major axes of subhalos point toward the center of the host halo (e.g., Barber et al. 2015). We sample from the priors folded with a normal distribution on the observed ellipticity with width $\sigma_{\epsilon}=0.05$ (using emcee; Foreman-Mackey et al. 2013) and for each sample compute the mass within the half-light ellipsoid from Equation (6). The results for a range of observed ellipticities are shown in Figure 5. We show the mass estimates over the spherical mass estimator using the "circularized" radius. We see that using the spherical mass estimator in this way reproduces the mass within the half-light ellipsoid over the full range of ellipticities. ${ }^{3}$ The uncertainty in the estimator increases with increasing ellipticity but is only $\sim 10 \%-20 \%$ for $\epsilon \sim 0.4$ (a typical dSph flattening). There is the tendency for the mass within the half-light ellipsoid to be overestimated for large $\epsilon$, but only by $\sim 5 \%$. We also show the

\footnotetext{
3 We note that a similar observation was made by Laporte et al. (2013), who found that the variations of $R_{\mathrm{h}}$ and $\left\langle\sigma_{\text {los }}^{2}\right\rangle$ with triaxiality compensated each other to give an unbiased mass estimate.
}

distribution of $f_{r} / \sqrt{1-\epsilon}$ for each prior assumption (i.e., the ratio of the size of the ellipsoid to the $R_{\mathrm{h}} \sqrt{1-\epsilon}$ approximation). For the uniform prior, this ratio is unity (within $\sim 10 \%-20 \%$ ) so the "size" of the dSphs are well approximated by $R_{\mathrm{h}} \sqrt{1-\epsilon}$. For the other two priors, the ratio increases with ellipticity as the intrinsic ellipsoids are on average more elongated along the LOS so larger than $R_{\mathrm{h}} \sqrt{1-\epsilon}$.

We have demonstrated that the mass within the half-light ellipsoid can be accurately estimated using the spherical mass estimator formulae. Although we do not know the shape or orientation of this half-light ellipsoid, we can say with confidence the mass within it. Therefore, we can accurately estimate the mass-to-light ratio using the mass within the halflight ellipsoid and half the total luminosity $L$. We conclude that using the spherical mass estimators (Walker et al. 2009; Wolf et al. 2010) with the "circularized" half-light radius produces accurate estimates of the mass-to-light ratio of dSphs, irrespective of flattening, provided the light and dark matter are stratified on the same self-similar concentric ellipsoids.

\section{CONCLUSIONS}

This Letter has answered the question: how should the mass of a flattened, dispersion-supported galaxy like a dwarf spheroidal be estimated? If the galaxy were spherical, then the answer is well established. Accurate mass estimators depending on the observable half-light radius and the velocity dispersion of the stars have been devised by a number of investigators (Walker et al. 2009; Wolf et al. 2010; Amorisco \& Evans 2012; Campbell et al. 2016).

We have shown how to modify the spherical mass estimators so that they work for flattened systems in which the light and dark matter are stratified on the same concentric self-similar ellipsoids. This represents a limiting case as simulations indicate the dark-matter distribution is in fact rounder than the light (Abadi et al. 2010; Zemp et al. 2012) due to baryonic feedback effects, particularly for the more massive dSphs. The modifications require knowledge of the intrinsic shape and alignment of the triaxial figure and reproduce the mass within ellipsoids by deprojecting the half-light radius and LOS velocity dispersion. The resulting mass estimates are independent of details of the radial profile and are as accurate as the corresponding spherical formulae.

This would be of little use if we require knowledge of intrinsic properties. However, we have also shown that, when averaging over triaxial configurations that are consistent with the observed ellipticity $\epsilon$, major-axis half-light length $R_{\mathrm{h}}$, and LOS velocity dispersion, the mass within the half-light ellipsoid is well approximated by the spherical mass estimate using the "circularized" half-light radius of $R_{\mathrm{h}} \sqrt{1-\epsilon}$. The scatter in the estimate increases with ellipticity but is only $10 \%-20 \%$ for $\epsilon \sim 0.6$. In turn, this observation implies that mass-to-light ratios using spherical estimators, together with a luminosity of $L_{1 / 2}=L / 2$, are accurate and insensitive to the flattening of the $\mathrm{dSph}$. This therefore provides a surprisingly simple, flexible, and effective way to account for the effects of flattening.

\section{REFERENCES}

Abadi, M. G., Navarro, J. F., Fardal, M., Babul, A., \& Steinmetz, M. 2010, MNRAS, 407, 435

Amorisco, N. C., \& Evans, N. W. 2012, MNRAS, 419, 184 
Barber, C., Starkenburg, E., Navarro, J. F., \& McConnachie, A. W. 2015, MNRAS, 447, 1112

Binney, J., \& Tremaine, S. 2008, Galactic Dynamics (2nd ed.; Princeton, NJ: Princeton Univ. Press)

Campbell, D. J. R., Frenk, C. S., Jenkins, A., et al. 2016, arXiv:1603.04443

Foreman-Mackey, D., Conley, A., Meierjurgen Farr, W., et al. 2013, emcee: The MCMC Hammer, Astrophysics Source Code Library, ascl:1303.002

Koposov, S. E., Belokurov, V., Torrealba, G., \& Evans, N. W. 2015, ApJ, 805,130

Laporte, C. F. P., Walker, M. G., \& Peñarrubia, J. 2013, MNRAS, 433, L54
Roberts, P. H. 1962, ApJ, 136, 1108

Sánchez-Janssen, R., Ferrarese, L., MacArthur, L. A., et al. 2016, ApJ, 820, 69

Sanders, J. L., Evans, N. W., Geringer-Sameth, A., \& Dehnen, W. 2016, PhRvD, 94, 063521

Walker, M. G., Mateo, M., Olszewski, E. W., et al. 2009, ApJ, 704, 1274

Weijmans, A.-M., de Zeeuw, P. T., Emsellem, E., et al. 2014, MNRAS, 444, 3340

Wolf, J., Martinez, G. D., Bullock, J. S., et al. 2010, MNRAS, 406, 1220

Zemp, M., Gnedin, O. Y., Gnedin, N. Y., \& Kravtsov, A. V. 2012, ApJ, 748,54 